\title{
Optimization of Quercetin loaded Palm Oil Ester Based Nanoemulsion Formulation for Pulmonary Delivery
}

\author{
Noor Hafizah Arbain ${ }^{1,2}$, Norazlinaliza Salim ${ }^{1,2}$, Wong Tin Wui ${ }^{3}$, Mahiran Basri, ${ }^{1,2}$, and \\ Mohd Basyaruddin Abdul Rahman, 2,* \\ ${ }^{1}$ Integrated Chemical Biophysics Research, Faculty of Science, Universiti Putra Malaysia, 43400 UPM Serdang, Selangor, MALAYSIA \\ ${ }^{2}$ Department of Chemistry, Faculty of Science, Universiti Putra Malaysia, 43400 UPM Serdang, Selangor, MALAYSIA \\ ${ }^{3}$ Non-Destructive Biomedical and Pharmaceutical Research Centre, iPROMISE, Universiti Teknologi MARA, 42300, Puncak Alam, Selangor, \\ MALAYSIA
}

\begin{abstract}
In this research, the palm oil ester (POE)- based nanoemulsion formulation containing quercetin for pulmonary delivery was developed. The nanoemulsion formulation was prepared by high energy emulsification method and then further optimized using D-optimal mixture design. The concentration effects of the mixture of POE:ricinoleic acid (RC), ratio 1:1 (1.50-4.50 wt.\%), lecithin (1.50-2.50 wt.\%), Tween 80 (0.50-1.00 wt.\%), glycerol (1.50-3.00 wt.\%), and water (88.0-94.9 wt.\%) towards the droplet size were investigated. The results showed that the optimum formulation with 1.50 wt.\% POE:RC, 1.50 wt.\% lecithin, 1.50 wt.\% Tween 80, 1.50 wt.\% glycerol and $93.90 \%$ water was obtained. The droplet size, polydispersity index (PDI) and zeta potential of the optimized formulation were $110.3 \mathrm{~nm}, 0.290$ and -37.7 $\mathrm{mV}$, respectively. The formulation also exhibited good stability against storage at $4^{\circ} \mathrm{C}$ for 90 days. In vitro aerosols delivery evaluation showed that the aerosols output, aerosols rate and median mass aerodynamic diameter of the optimized nanoemulsion were $99.31 \%, 0.19 \mathrm{~g} / \mathrm{min}$ and $4.25 \mu \mathrm{m}$, respectively. The characterization of physical properties and efficiency for aerosols delivery results suggest that POE- based nanoemulsion containing quercetin has the potential to be used for pulmonary delivery specifically for lung cancer treatment.
\end{abstract}

Key words: palm oil ester, quercetin, nanoemulsion, aerosols, pulmonary delivery

\section{INTRODUCTION}

Cigarette smoking is known as the leading cause of pulmonary diseases such as lung cancer and chronic obstructive pulmonary disease (COPD). Approximately $90 \%$ of lung cancer and $80 \%$ of COPD cases are linked to cigarette smoking. In fact, lung cancer was one of the primary causes of cancer-related death worldwide. Between 2010 and 2014, smoking caused nearly half a million premature deaths every year, more than $87 \%$ of all lung cancer deaths and $61 \%$ of all pulmonary deaths ${ }^{1)}$.

Chemotherapeutic methods using natural molecules are becoming increasingly used to inhibit or reverse carcinogenesis $^{2,3)}$. Quercetin, is a well-known flavonoid distributed in plants with desired therapeutic benefits and disease preventing properties, for example, anti- tumor ${ }^{4-7)}$. Studies reported that quercetin could inhibit proliferation of multiple cancer cell types, including lung cancer, colon cancer, prostate carcinoma, and pancreatic tumor ${ }^{8,9)}$. However, quercetin has low solubility in water, thus it has limited bioavailability $^{10)}$. Quercetin contains five hydroxyl groups that reflect its biological activities. The hydrophobic nature of quercetin is due to the existence of benzene ring and strong intramolecular H-bonding which renders its ability to form strong complex's interaction, thus affecting its bioavailability $^{8)}$. Various approaches have been introduced to enhance the solubility of quercetin ${ }^{11-13)}$. Among the delivery system, nanoemulsions have promising delivery system that could enhance the solubility with favorable characteristics, for example ease of preparation, thermodynamically stable, increase of bioavailability, high loading capacity and encapsulation efficiency ${ }^{11,14)}$.

Nanoemulsions have the advantages such as being apparently transparent or translucent, ease of preparation, thermodynamic stability and increased surface area due to very fine droplet size which is in between 20 and $200 \mathrm{~nm}^{15)}$. Nanoemulsions have the potential to deliver proteins as

\footnotetext{
*Correspondence to: Mohd Basyaruddin Abdul Rahman, Department of Chemistry, Faculty of Science, Universiti Putra Malaysia, 43400 UPM Serdang, Selangor, MALAYSIA

E-mail: basya@upm.edu.my

Accepted February 27, 2018 (received for review November 28, 2017)

Journal of Oleo Science ISSN 1345-8957 print / ISSN 1347-3352 online

http://www.jstage.jst.go.jp/browse/jos/ http://mc.manusriptcentral.com/jjocs
} 
well as other new (or traditional) active drug compounds to the lungs because of their high solubilizing and drug loading efficiency ${ }^{16)}$. Furthermore, they have the ability to enhance pulmonary deposition and retention for prolonged periods in the lung tissues ${ }^{17)}$. The commonly used materials in the preparation of nanoemulsion are biocompatible. Palm oil derivatives such as palm oil ester (POE) have been used as penetration enhancers and nanocarriers for various drugs for example ibuprofen ${ }^{18)}$ and ketoprofen ${ }^{19)}$. Besides, they also have a wide variety of applications such as in food, agrochemicals and cosmeceuticals ${ }^{20)}$. POE for pharmaceutical application should be explored and expanded due to its favorable properties, for example biodegradable, stable at high temperature and resistant to oxidation whereby it can be a good oil phase as a therapeutic carrier for hydrophobic drugs. The POE can be enzymatically synthesized from palm oil with fatty alcohols. The compositions of POE including oleyl laurate, oleyl myristate, oleyl palmitate,oleyl stearate, oleyl oleate and oleyl linoleate ${ }^{21)}$. Palm oil contains the main health promoting chemical such as tocopherols, tocotrienols (vitamin E) and carotenoids that are useful in the prevention of cancer, pulmonary diseases and anti-inflammatory ${ }^{22}$. To the best of our knowledge, there is no existing study which has been reported on POE in preparation of nanoemulsions for pulmonary drug delivery.

The D-optimal mixture design was used for the optimization of mixture compositions to obtain desirable properties. In mixture experiments, the measured response was not dependent on the amount of the mixture but it was assumed to depend only on the relative proportions of the ingredients or components in the mixture. The total sum of all ingredients equaled to $100 \%$ and the experimental region and model making were influenced by each independent variable in the mixture design approach. This technique helped to minimize the number of experiments to be carried out and determine the interaction statistically to overcome the shortcoming of the other method. Nowadays, the D- optimal mixture design is used in several studies for optimization of mixture compositions for pharmaceuticals $^{23)}$ and cosmeceuticals nanoformulation ${ }^{24,25)}$.

This study focused on the optimization of POE- based nanoemulsion containing quercetin using the D- optimal mixture design in order to obtain nano droplet size of emulsion. The effects of the components and relationships among the factors towards the droplet size were investigated. The physicochemical properties and efficiency for pulmonary delivery of the optimized nanoemulsion were also determined.

\section{MATERIALS AND METHODS}

\subsection{Materials}

Palm oil ester (POE) was prepared in our laboratory through enzymatic alcoholysis of palm oil with oleyl alcohol using Lipozyme RM IM as the catalyst ${ }^{21)}$. Quercetin (purity $>90 \%$ ) and ricinoleic acid (RC) were purchased from Sigma-Aldrich (Germany). Tween 80 (polyoxyethylene sorbitan monooleate) was purchased from Merck (Germany). Pure soy bean lecithin was purchased from Lipoid GmbH (Ludwigshafen, Germany). Glycerol was purchased from JT Baker (Philipsburg, NJ, USA). Water was deionized using a Milli-Q filtration system, EMD Millipore(Billerica, MA, USA).

\subsection{Selection of oils}

The solubility of quercetin in the mixture of POE with different types of oils (olive and safflower seed) and different fatty acids (ricinoleic acid, linoleic acid and oleic acid) at the ratio of 1:1 (wt. \%) were determined. An access amount of quercetin was added into $3 \mathrm{~g}$ oils mixture. The solution was kept under moderate magnetic stirring (IKA, China)for $24 \mathrm{~h}$. The sample was then centrifuged (Hettich Zentrifugen, Germany) at $4000 \mathrm{rpm}$ for $15 \mathrm{~min}$ in order to separate undissolved quercetin. An aliquot of the supernatant was diluted with ethanol and the concentration of quercetin was determined using High Performance Liquid Chromatography- Ultra-violet (HPLC- UV) at wavelength of $372 \mathrm{~nm}$ based on a standard curve of known quercetin concentration. The HPLC system (Waters Corporation, Milford, MA) was equipped with a reverse phase C18 column (Symmetry C18 column - $3.5 \mu \mathrm{m}, 4.6 \times 75 \mathrm{~mm}$ ) and UV detector. The mobile phase ratio of acetonitrile and water was 45:55 at a flow rate of $1 \mathrm{ml} / \mathrm{min}$ at ambient temperature. A series of standard quercetin solutions was prepared in ethanol by a serial dilution at the concentration of between $20 \mu \mathrm{g} / \mathrm{mL}$ to $100 \mu \mathrm{g} / \mathrm{mL}$.

\subsection{Preparation of nanoemulsion}

Quercetin $(0.05 \%$ w/w $)$ was dissolved in oil phase (POE:RC, 1:1 ratio (wt.\%)) containing lecithin (2.00 wt. \%) at $40^{\circ} \mathrm{C}$. After quercetin was completely dissolved, Tween 80 (1.00 wt. \%) was added into the oil phase. The oil phase was added dropwise into a beaker containing aqueous phase (glycerol and water). The solution was stirred for 30 min using an overhead stirrer(IKA@ RW 20 Digital, Nara, Japan) at $300 \mathrm{rpm}$. The emulsion obtained was further subjected to high shear homogenizer at 10,000 rpm for 15 $\min$.

\subsection{Selection of surfactants}

The selection of surfactants was carried out by formulation of emulsion. The experiment was repeated using different surfactants (Cremophor EL (CrEL) and polyethylene glycol 400 (PEG 400). The formulation was analyzed with 
Table 1 Restrictions of component proportions (\%).

\begin{tabular}{cccc}
\hline \multirow{2}{*}{ Symbol } & \multirow{2}{*}{ Variables } & \multicolumn{2}{c}{ Range (wt. \%) } \\
\cline { 3 - 4 } & & Lower & Higher \\
\hline $\mathrm{A}$ & POE: RC & 1.50 & 4.50 \\
$\mathrm{~B}$ & Lecithin & 1.50 & 2.50 \\
$\mathrm{C}$ & Tween80 & 0.50 & 1.50 \\
$\mathrm{D}$ & Glycerol & 1.50 & 3.00 \\
$\mathrm{E}$ & Water & 88.50 & 94.90 \\
\hline
\end{tabular}

respect to the droplet size and polydispersity index (PDI).

\subsection{Experimental design}

D-optimal mixture design was used for the optimization of composition. The results were statistically analyzed using Design-Expert, Version 7.0 (Stat-Ease Inc., Minneapolis, USA) and Statistica, Version 12 (Statsoft Inc., Tulsa, USA). It was chosen for modeling and evaluating the effects of the five components on the droplet size of nanoemulsion. For a minimum number of experiments, this design supplies maximum amount of acquired information. Moreover, the optimum composition with desirable criteria can be numerically or graphically determined as the results are represented as the model ${ }^{23)}$. The five factor of D-optimal design mixture was utilized to investigate the effect of POE:RC (A), lecithin (B), Tween 80 (C), glycerol(D) and water $(\mathrm{E})$ on response variable (droplet size). The lower and higher limit of the independent variables were determined based on the data obtained from preliminary study and the water limit was determined by the software. In this work, the minimum and maximum proportions of these components were listed in Table 1. By following the D-optimal mixture design, the composition of each run was carried out in a randomized order so that the effect of the unexplained variability on the actual response caused by extraneous factor could be minimized ${ }^{26)}$. The contour and three dimensional surface graphs were plotted to show the effect of independent variables to response variable. The contour and three dimensional surface graphs for droplet size were used to interpret the significant interaction $(p<$ 0.05) effect of variables as a function of independent variables. The adequacy of the predicted response values in validation set was done by verification of the final model. The experimental and predicted values of droplet size of several additional randomized formulations were compared to evaluate the validity of the model.

\subsection{Statistical analysis}

Analysis of variance (ANOVA) and $\mathrm{R}^{2}$ (coefficient of determination) were carried out to investigate the significant differences among the independent variables. In order to get a good final reduced model, $p$ value must be significant $(p<0.05)$ and value of $\mathrm{R}^{2}$ higher than 0.9 is considered as model with very high correlation.

\subsection{Verification of models}

By preparing a few random formulations to validate the obtained models, the actual and theoretical prediction values were compared quantitatively. This is the most important step to make sure the adequacy of the final reduced models. The recommended optimum composition was also carried out to verify the predicted optimum values from the model. The response obtained was compared to the predicted values by calculating the residual standard error as in Equation 1:

$$
\begin{aligned}
& \text { Residual standard error (\% RSE) } \\
& =\frac{\text { Experimental value }- \text { Predicted value }}{\text { Predicted value }} \times 100 \\
& \text { Equation } 1
\end{aligned}
$$

\subsection{Characterization of the optimized nanoemulsion for- mulation}

2.8.1 Droplet size and polydispersity index

The droplet size and polydispersity index(PDI) of the prepared nanoemulsions were measured using Zetasizer (Nano ZS, Malvern Instrument Ltd., UK) at room temperature $\left(25^{\circ} \mathrm{C} \pm 0.5^{\circ} \mathrm{C}\right)$ using dynamic light scattering technique, scattered at an angle of $173^{\circ}$. For the measurement, the samples were diluted with deionized water (1:200) and injected into the sample cell. Intensity distribution was used for the measurement of mean average (z-average) droplet size. The measurement was repeated three times. 2.8.2 Zeta potential

Zeta potential analysis was measured using Zetasizer (Nano ZS, Malvern Instrument Ltd., UK) at room temperature $\left(25^{\circ} \mathrm{C} \pm 0.5^{\circ} \mathrm{C}\right)$. For the measurement, the samples were diluted with deionized water. The calculation of zeta potential was done based on measurement of the electrophoretic mobility of dispersed particles in a charged field.

2.8.3 Stability study

Stability study was carried out to observe any physical instability of nanoemulsion. The formulation was put in a container and was stored at $4{ }^{\circ} \mathrm{C}$ for 90 days. To test the stability of the samples, it was centrifuged at 4, $500 \mathrm{rpm}$ for 15 minutes. The physical appearances of the formulation were observed to see whether there were any precipitates formed.

2.8.4 In vitro evaluation of aerosol delivery of nanoemulsion

The aerodynamic and inhalation characteristics of the nanoemulsion were measured using an eight stage Andersen cascade impactor (ACI). The ACI was first assembled from filter to stage "0" with attached induction port that was clamped and sealed together with FDA- approved silicone rubber O-rings. The ACI was connected at the other end to a vacuum source. Each of the nanoemulsion $(3 \mathrm{~mL})$ 
was loaded in the medication compartment of an OMRON nebulizer and nebulized into the cascade impactor at a flow rate of $15 \mathrm{~L} / \mathrm{min}^{27)}$. Nebulization was commenced to dryness on continuous mode. Following dosage emission, each plate of ACI along with 'throat', mouth piece, induction port and filter was washed with ethanol into separate glass scintillation vials. The respective drug content of the washing solutions was then assayed using HPLC- UV at wavelength of $372 \mathrm{~nm}$. The data was then processed to determine the aerosolization and inhalation characteristics. Mass median aerodynamic diameter (MMAD) was obtained from the cumulative distribution curve at the $50 \%$ point ${ }^{28)}$. Aerosol output (\%) was determined by calculating the weight difference of the nebulizer before and after nebulization. The time required to reach "dryness" status was recorded and mass output rate was accordingly calculated as $\mathrm{g} / \mathrm{min}$.

\section{RESULTS AND DISCUSSION}

\subsection{The selection of oils}

Vegetable oils (olive and safflower seed) and fatty acids (ricinoleic, oleic and linoleic) were chosen as excipient due to their biocompatibility and low toxicity. Moreover, long chain fatty acid lipids have also been reported to form stable nanoemulsion without precipitation ${ }^{29)}$. The major constituents of palm oil ester (POE) were palmitic and oleic acids in which was reported to enhance the absorption of active molecules through membrane ${ }^{21)}$. Figure 1 shows that the solubility of quercetin was found to be highest in the mixture of POE: RC. This may be attributed to the polarity of the quercetin that favors their solubilization in more polar oils. Similar study has been reported in previous finding which they found that the solubility of the drug was dependent on the polarity of the oils ${ }^{30)}$. The polarity of the oils increased according to the order of olive and safflower seed oil (nonpolar) $<$ oleic and linoleic acid (unsaturated fatty acid) $<$ ricinoleic acid (hydroxyl group). Ricinoleic acid was more polar compared to the others due to the present of hydroxyl functional group at carbon- 12. However, solubility of QT in the olive oil, safflower seed oil, oleic acid and linoleic acid were in similar range, since they have similar fatty acid composition (18 carbon chain). Hence, the mixture of POE: RC was chosen as an oil phase for preparation of nanoemulsion containing quercetin in this study.

\subsection{The selection of surfactants}

In this study, a mixture of lecithin and surfactants with naturally derived phospholipids were chosen because of their biodegradability, biocompatibility and stability to many applications. Table 2 shows the droplet size of quercetinloaded nanoemulsion using the mixture of lecithin with surfactants of Tween 80, Chremophor EL, and PEG 400. The

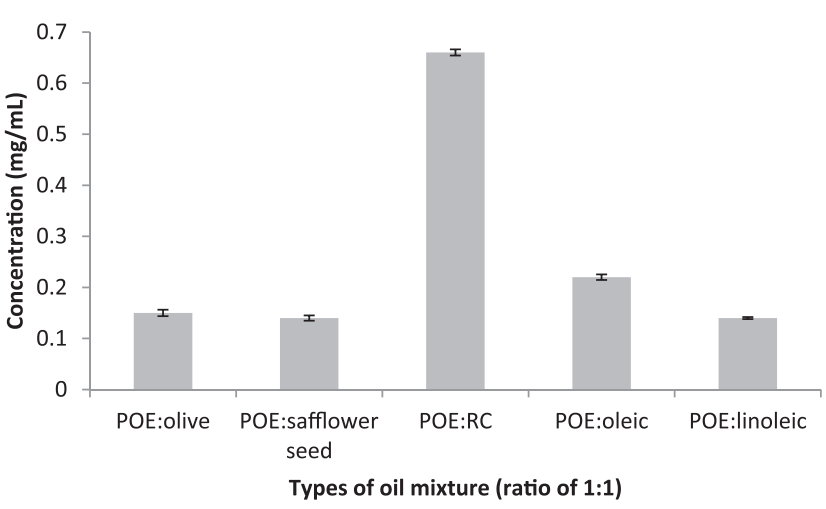

Fig. 1 Different concentration of quercetin in various oil mixtures.

Table 2 The droplet size and PDI values of nanoemulsions based different mixture of surfactant.

\begin{tabular}{lcc}
\hline Mixture of surfactant & Droplet size $(\mathrm{nm})$ & PDI \\
\hline Lecithin: T80 & $131.5 \pm 1.70$ & $0.258 \pm 0.004$ \\
Lecithin: CrEL & $137.3 \pm 1.26$ & $0.260 \pm 0.002$ \\
Lecithin: PEG 400 & $184.4 \pm 2.87$ & $0.346 \pm 0.033$ \\
\hline
\end{tabular}

lower PDI values for all formulations showed the uniform distribution of droplet size. The smaller droplet size of nanoemulsion was obtained when lecithin was mixed with Tween 80 followed by Chremophor EL, and PEG 400. The differences observed in their emulsifying ability might be attributed to the molecular weight of the surfactants ${ }^{31}$. The molecular weight of Tween $80(1310 \mathrm{~g} / \mathrm{mol})$ is the highest compared to Chremophor EL (801-900 g/mol) and PEG 400 (380-420 g/ $\mathrm{mol}$ ). The larger molecular weight and longer chain length of the surfactant improved the encapsulation efficiency of drugs $^{32)}$. This explains why the mixture of lecithin and Tween 80 formed the smallest droplet size compared to other surfactants due to better link to encapsulate the oil droplet. Furthermore, both of them had similarities of long oleic acid structures, which enabled them to have better compatibility and form a homogeneous interface that prevented them from aggregation.

\subsection{Fitting the models}

Table 3 shows the values obtained from the experiment were in agreement with the predicted values. A linear model was fitted by the D- optimal design. The final equations for the models describing the droplet size can be written as Equation 2:

Droplet Size:

$$
\mathrm{Y}=+205.43 \mathrm{~A}+146.48 \mathrm{~B}-7.03 \mathrm{C}+126.38 \mathrm{D}+131.97 \mathrm{E}
$$

Equation 2

Analysis of variance (ANOVA) was used to evaluate the coefficient significance of the models (Table 4). The model 
Table 3 Predicted and actual values of droplet size of nanoemulsions obtained from D-optimal mixture experimental design.

\begin{tabular}{cccccccc}
\hline \multirow{2}{*}{ Run No. } & POE:RC & Lecithin & Tween 80 & Glycerol & Water (E), & \multicolumn{2}{c}{ Droplet size (nm) } \\
\hline 1 & (A), (wt.\%) & (B), (wt.\%) & $($ C), (wt.\%) & (D), (wt.\%) & $($ wt. $\%)$ & Actual & Predicted \\
\hline 2 & 4.500 & 1.928 & 1.500 & 3.000 & 89.023 & 145.90 & 144.26 \\
3 & 1.626 & 2.500 & 1.500 & 1.615 & 92.708 & 114.20 & 114.01 \\
4 & 1.794 & 2.335 & 1.499 & 3.000 & 91.323 & 114.30 & 114.36 \\
5 & 4.159 & 1.500 & 0.500 & 2.026 & 91.765 & 161.40 & 161.81 \\
6 & 4.197 & 1.500 & 1.407 & 1.502 & 91.344 & 142.50 & 143.15 \\
7 & 4.498 & 2.500 & 0.531 & 1.584 & 90.837 & 166.90 & 167.63 \\
8 & 4.500 & 1.500 & 0.910 & 2.715 & 90.325 & 156.40 & 156.25 \\
9 & 3.074 & 2.087 & 0.501 & 3.000 & 91.288 & 150.80 & 149.90 \\
10 & 2.706 & 1.500 & 0.500 & 1.568 & 93.675 & 146.40 & 145.64 \\
11 & 4.499 & 2.500 & 1.499 & 1.908 & 89.545 & 147.50 & 146.50 \\
12 & 4.498 & 2.500 & 0.531 & 1.584 & 90.837 & 167.70 & 167.63 \\
13 & 4.500 & 1.928 & 1.500 & 3.000 & 89.023 & 144.90 & 144.26 \\
14 & 1.524 & 2.500 & 0.507 & 2.338 & 93.080 & 134.40 & 133.61 \\
15 & 1.500 & 2.177 & 1.284 & 2.614 & 92.375 & 115.30 & 115.64 \\
16 & 4.197 & 1.500 & 1.407 & 1.502 & 91.344 & 142.90 & 143.15 \\
17 & 1.524 & 2.500 & 0.507 & 2.338 & 93.080 & 133.10 & 133.61 \\
18 & 4.499 & 2.500 & 0.877 & 2.998 & 89.077 & 157.50 & 158.95 \\
19 & 1.626 & 2.500 & 1.500 & 1.615 & 92.708 & 114.45 & 114.01 \\
20 & 2.381 & 1.863 & 1.500 & 2.136 & 92.071 & 120.85 & 120.72 \\
21 & 2.040 & 1.501 & 0.501 & 3.000 & 92.908 & 136.70 & 136.82 \\
22 & 1.500 & 1.500 & 1.086 & 2.621 & 93.244 & 116.60 & 118.38 \\
23 & 3.122 & 2.500 & 1.500 & 3.000 & 89.828 & 129.80 & 129.85 \\
24 & 3.577 & 2.252 & 1.500 & 2.029 & 90.591 & 134.10 & 135.31 \\
25 & 3.235 & 2.114 & 0.952 & 1.500 & 92.149 & 143.20 & 143.37 \\
\hline & 1.500 & 1.500 & 0.500 & 1.500 & 94.949 & 133.00 & 131.98 \\
\hline
\end{tabular}

had large $F$-values and small $p$-values indicating significant effects on the respective response variable ${ }^{26)}$. As for the $p$-value, it is considered as significant when the values are less than $0.0500^{24)}$. The final reduced model had low standard deviation $( \pm 0.89)$, high estimated regression coefficients, $R^{2}(0.9976)$ and adjusted $R^{2}(0.9971)$. The results show that more than $99 \%$ of the response variations of the independent variables could be described by the models as a function of the main composition.

\subsection{D-optimal analysis}

Figures 2 and 3 show that the droplet size of emulsion increases as the amount of oil (POE: RC) increases. Increasing amount of oil phase resulted in increasing viscosity of the dispersed phase which causes an increase in flow resistance and restriction on the droplet break-up process thus causing formation of larger particles ${ }^{23)}$. On the other hand,
Table 4 Analysis of variance (ANOVA) for the linear models derived by D-optimal mixture design.

\begin{tabular}{crcc}
\hline \multirow{2}{*}{ Source } & \multicolumn{3}{c}{ Droplet size $(\mathrm{nm})$} \\
\cline { 2 - 4 } & $\begin{array}{c}\text { Mean } \\
\text { square }\end{array}$ & $F$ - value & $p$ - value \\
\hline Model & 1638.19 & 2073.62 & $<0.0001$ \\
Linear Mixture & 1638.19 & 2073.62 & $<0.0001$ \\
Residual & 0.79 & - & - \\
Lack of fit & 0.93 & 2.63 & 0.1453 \\
Pure error & 0.36 & - & - \\
\hline
\end{tabular}




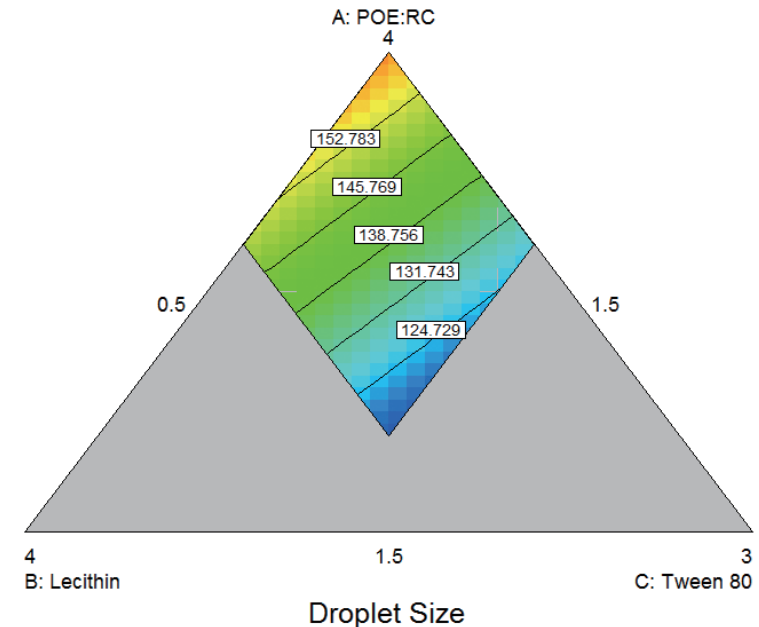

Fig. 2 Contour plot showing the interaction effect between three variables (POE: RC, lecithin, Tween 80) on the response (droplet size). Two variables were kept constant (glycerol and water).

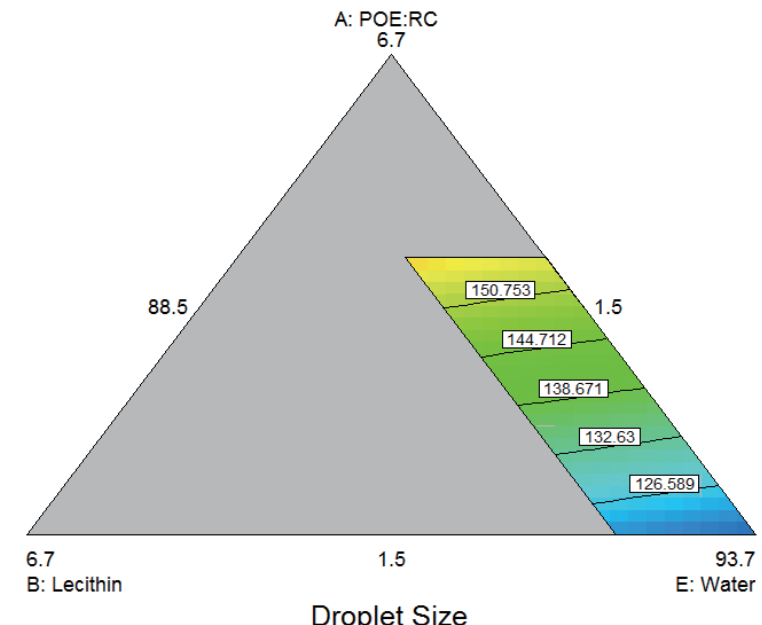

Fig. 3 Contour plot showing the interaction effect between three variables (POE: RC, lecithin, water) on the response (droplet size). Two variables were kept constant (glycerol and Tween 80).

Table 5 Validation set for four different formulations of nanoemulsion- containing quercetin.

\begin{tabular}{|c|c|c|c|c|c|c|c|c|}
\hline & \multicolumn{5}{|c|}{ Components (wt. \%) } & \multicolumn{3}{|c|}{ Droplet Size (nm) } \\
\hline & POE:RC & Lecithin & Tween 80 & Glycerol & Water & $\begin{array}{c}\text { Actual } \\
\text { Value }\end{array}$ & $\begin{array}{c}\text { Predicted } \\
\text { Value }\end{array}$ & RSE (\%) \\
\hline & 3.30 & 2.00 & 1.00 & 2.25 & 91.40 & 142.00 & 142.17 & 0.12 \\
\hline Validation & 3.40 & 2.00 & 1.00 & 2.25 & 91.30 & 142.40 & 143.31 & 0.64 \\
\hline \multirow[t]{2}{*}{ Set } & 3.00 & 2.00 & 1.20 & 2.25 & 91.50 & 134.30 & 134.45 & 0.11 \\
\hline & 3.00 & 2.00 & 1.00 & 2.40 & 91.55 & 138.20 & 138.63 & 0.31 \\
\hline
\end{tabular}

as the amount of surfactant (lecithin and Tween 80)increased, a decrease in the particle size values was observed. This could be due to the presence of more emulsifier that could cover the new droplet surfaces formed during homogenization and interfacial tensions lowered between oil and water ${ }^{24)}$.

In Fig. 3, increasing the water composition seemed to decrease the droplet size of emulsion. Increasing amount of water caused the emulsion to be less viscous. As the viscosity decreased, the formation of larger droplet size could be prevented due to decrease in flow resistance and increase in droplet disruption efficiency.

\subsection{Verification of the model}

Table 5 shows a good indication on the fitness of model generated as all the results showed no significant difference between the actual and predicted values. The values of formulation result were determined by the percentage of residual standard error (\% RSE) which were less than \pm $5.00 \%$. The results confirmed the adequacy of the model and indicated excellent fitness of the model generated as the actual values were found to be quite close to the predicted values.

\subsection{Optimization of D-optimal mixture design for nano- emulsion}

By determining the optimization constraint and evaluating the interaction effect between independent variables, the optimized quercetin nanoemulsion was prepared based on smaller droplet size of nanoemulsion. Using this approach, optimized formulation obtained was 1.5\% of POE:RC, $1.5 \%$ of lecithin, 1.5\% of Tween 80, 1.5\% of glycerol and 93.95\% of water.The predicted value was $110.4 \mathrm{~nm}$. This value was close to the experimental value, which was $110.3 \mathrm{~nm}$. The\% RSE was small $(0.109 \%)$ and insignificant. Hence, the model obtained was significant and the optimized formulation can be used for further characterization.

\subsection{Characterization of the optimized nanoemulsion con- taining quercetin}

The droplet size and PDI of nanoemulsion of $110.3 \pm 0.35$ $\mathrm{nm}$ and $0.290 \pm 0.005$, were obtained. PDI value described the uniformity of particle distribution in the emulsion system. Lower PDI value of the optimized formulation indicated a narrow size distribution of the droplet. The zeta potential obtained was $-37.7 \pm 0.99 \mathrm{mV}$. The stability of nanoparticles in suspension was greatly improved due to 
the increasing repulsive forces against flocculation and coalescence predominant between droplets in the nanoemulsions systems as the zeta potential values are $>+30 \mathrm{mV}$ or $<-30 \mathrm{mV}^{33)}$. The nanoemulsion remained physically stable with no precipitation or sedimentation observed during storage for 90 days at $4^{\circ} \mathrm{C}$.

The aerosols output for the optimum formulation was $99.31 \pm 0.01 \%$. The aerosols output refers to the amount of fluid successfully delivered by the nebulizer. A very high aerosols output means low residual volumes in the nebulizer. Previous study demonstrated that the nanoemulsion in the study produced aerosols output of approximately $90 \%{ }^{17)}$. The aerosols rate for the optimized formulation was $0.19 \pm$ $0.01 \mathrm{~g} / \mathrm{min}$. The aerosols output rate for the Omron Micro Air was between $0.02 \mathrm{~g} / \mathrm{min}-0.3 \mathrm{~g} / \mathrm{min}^{34,35)}$.

Aerosol particles were also characterized by their mass median aerodynamic diameter(MMAD). Particles with aerosol droplets between 1 and $5 \mu \mathrm{m}$ are optimal for deposition in the lower airway, and are deposited largely by inertial impaction with airway structures. Particles with aerosol droplets greater than $5 \mu \mathrm{m}$ are deposited largely in the oropharynx, while those less than $1 \mu \mathrm{m}$ being generally exhaled $^{28)}$. The MMAD for optimum formulation was $4.25 \pm$ $0.38 \mu \mathrm{m}$ which indicated that the formulated nanoemulsion showed suitability for aerosolization to be inhaled into the lung since their MMAD value was in the range of MMAD that was suitable for inhalation.

\section{CONCLUSION}

D-optimal mixture experiment design proved to be effective for composition optimization. This study demonstrated that quercetin nanoemulsions -based palm oil was optimized by combining five independent variables: POE:RC, lecithin, Tween 80 , glycerol and water. The actual value of the response obtained from experimental results were in good agreement with predicted values as residual standard error (RSE) for the optimal composition was $0.11 \%$. The optimum formulation of $1.50 \mathrm{wt} . \%$ POE:RC, $1.50 \mathrm{wt} . \%$ of lecithin, 1.50 wt.\% of Tween 80, 1.50 wt.\% of glycerol and 93.90 wt. \% of water with droplet size of $110.3 \mathrm{~nm}$, PDI value of 0.290 and zeta potential of $-37.7 \mathrm{mV}$ were obtained while remaining physically stable upon storage for 90 days at $4^{\circ} \mathrm{C}$. In vitro evaluation of aerosols delivery of nanoemulsion demonstrated that the optimized nanoemulsion showed suitability for pulmonary drug delivery.

\section{ACKNOWLEDGMENTS}

The financial assistances received from Ministry of Higher Education Malaysia (MOHE) through MyBRAIN 15 for Arbain N.H. This work was supported by Universiti
Malaya, LRGS NanoMITe-Ministry of Higher Education, Malaysia (RU029-2014/5526306).

\section{References}

1) World Health Organization (WHO). International Agency for Research on Cancer. GLOBOCAN 2012: Estimated Cancer Incidence, Mortality and Prevalence Worldwide in 2012. Accessed April 12, 2015.

2) Gupta, S.C.; Kim, S.H.; Prasad, S.; Aggarwal, B.B. Regulation of survival, proliferation, invasion, angiogenesis, and metastasis of tumor cells through modulation of inflammatory pathways by nutraceuticals. Cancer Metastasis Rev. 29, 405-434 (2010).

3) Kennedy, D.A.; Hart, J.; Seely, D. Cost effectiveness of natural health products: A systematic review of randomized clinical trials. Evid-Based Complement Altern Med. 6, 297-304 (2009).

4) Wu, T.H.; Yen, F.L.; Lin,L.T.; Tsai, T.R.; Lin, C.C.; Chamd, T.M. Preparation, physicochemical characterization, and antioxidant effects of quercetin nanoparticles. Int. J. Pharm. 346, 160-168(2008).

5) Schaab, M.R.; Barney, B.M.; Francisco, W.A. Kinetic and spectroscopic studies on the quercetin 2, 3-dioxygenase from Bacillus subtilis. Biochemistry 45, 10091016 (2006).

6) Kanadaswami, C.; Lee, L.T.; Lee, P.P.; Hwang, J.J.; Ke, F.C.; Huang, Y.T.; Lee, M.T. The antitumor activities of flavonoids. In Vivo 19, 895-909 (2005).

7) Comalada, M.; Camuesco, D.; Sierra, S.; Ballester, I.; Xaus, J.; Galvez, J.; Zarzuelo, A. In vivo quercitrin anti-inflammatory effect involves release of quercetin, which inhibits inflammation through down-regulation of the NF-kappaB pathway. Eur. J. Immunol. 35, 584$592(2005)$.

8) Nam, J.; Sharma, A. R.; Nguyen, L. T.; Chakraborty, C.; Sharma, G.; Lee, S.S. Review. Application of bioactive quercetin in oncotherapy: From nutrition to nanomedicine. Molecules 21, 108(2016).

9) Li, H.; Zhao, X.; Ma, Y.; Zhai, G.; Li, L.; Lou, H. Enhancement of gastrointestinal absorption of quercetin by solid lipid nanoparticles. J. Controlled Release. 133, 238-244 (2009).

10) Gao, Y.; Wang, Y.; Ma, Y.; Yu, A.; Cai, F.; Shao, W.; Zhai, G. Formulation optimization and in situ absorption in rat intestinal tract of quercetin-loaded microemulsion. Colloids Surf. B 71, 306-314(2009).

11) Aditya, N.P.; Macedo, A.S.; Doktorovova, S.; Souto, E.B.; Kim, S.; Chang, P.S.; Ko, S. Development and evaluation of lipid nanocarriers for quercetin delivery: A comparative study of solid lipid nanoparticles (SLN), nanostructured lipid carriers (NLC), and lipid nanoemulsions (LNE). LWT-Food Sci. Technol. 59, 115- 
121(2014)

12) Verma, N.K.; Crosbie-Staunton, K.; Satti, A.; Gallagher, S.; Ryan, K.B.; Doody, T.; Gun'ko, Y.K. Magnetic coreshell nanoparticles for drug delivery by nebulization. $J$. Nanobiotechnol. 11, 1(2013).

13) Pimple, S.; Manjappa, A.S.; Ukawala, M.; Murthy, R.S. PLGA nanoparticles loaded with etoposide and quercetin dihydrate individually: In vitro cell line study to ensure advantage of combination therapy. Cancer Nanotechnol. 3, 25-36 (2012).

14) Karadag, A.; Yang, X.; Ozcelik, B.; Huang, Q. Optimization of preparation conditions for quercetin nanoemulsions using response surface methodology. J. Agric. Food Chem. 61, 2130-2139(2013).

15) Solans, C.; Izquierdo, P.; Nolla, J.; Azemar, N.; GarciaCelma, M.J. Nano-emulsions. Current Opin. Colloid Interface Sci. 10, 102-110(2005).

16) Amani, A.; York, P.; Chrystyn, H.; Clark, B.J. Evaluation of a nanoemulsion-based formulation for respiratory delivery of budesonide by nebulizers. AAPS PharmSciTech. 11, 1147-1151 (2010).

17) Nasr, M.; Nawaz, S.; Elhissi, A. Amphotericin B lipid nanoemulsion aerosols for targeting peripheral respiratory airways via nebulization. Int. J. Pharm. 436, 611-616 (2012).

18) Salim, N.; Basri, M.; Rahman, M.B.A.; Abdullah, D.K.; Basri, H. et al. Phase behaviour, formation and characterization of palm-based esters nanoemulsion formulation containing ibuprofen. J. Nanomedic. Nanotechnol. 2, 113(2011).

19) Sakeena, M.H. F.; Muthanna, F.; Ghassan, Z.; Kanakal, M.M.; Elrashid, S.M.; Munavvar, S.; Azmin, M.N. Formulation and in vitro evaluation of ketoprofen in palm oil esters nanoemulsion for topical delivery. J. Oleo Sci. 59, 223-228(2010).

20) Teo, B.S.X.; Basri, M.; Zakaria, M.R.S.; Salleh,A.B.; Abdul Rahman, R.N.Z.R.; Rahman, M.B.A. A potential tocopherol acetate loaded palm oil esters-in-water nanoemulsions for nanocosmeceuticals. J. Nanobiotechnol. 8, 4(2010).

21) Keng, P.S.; Basri, M.; Zakaria, M.R.S.; Rahman, M.B.A.; Ariff, A.B.; Rahman, R.N.Z.A.; Salleh, A.B. Newly synthesized palm esters for cosmetics industry. Ind. Crop Prod. 29, 37-44(2009).

22) Rao, Narasinga. Bioactive phytochemicals in Indian foods and their potential in health promotion and disease prevention. Asia Pac. J. Clin. Nutr. 12, 9-22 (2003).

23) Masoumi, H.R.F.; Basri, M.; Samiun, W.S.; Izadiyan, Z.; Lim, C.J. Enhancement of encapsulation efficiency of nanoemulsion-containing aripiprazole for the treatment of schizophrenia using mixture experimental design. Int. J. Nanomedicine 10, 6469-6471(2015).

24) Samson, S.; Basri, M.; Fard Masoumi, H.R.; Karjiban,
R.A.; Malek, E.A. Design and development of a nanoemulsion system containing copper peptide by D- optimal mixture design and evaluation of its physicochemical properties. RSC Adv. 6, 17845-17856 (2016).

25) Che Sulaiman, I.S.; Basri, M.; Masoumi, H.R.F.; Asharia, S.E.; Ismail, M. Design and development of a nanoemulsion system containing extract of Clinacanthus nutans (L.) leaves for transdermal delivery system by D-optimal mixture design and evaluation of its physicochemical properties. RSC Adv. 6, 67378-67388 (2016).

26) Musa, S.H.; Basri, M.; Masoumi, H.R.F.; Karjiban, R.A.; Malek, E.A.; Basri, H; Shamsuddin, A.F. Formulation optimization of palm kernel oil esters nanoemulsionloaded with chloramphenicol suitable for meningitis treatment. Colloids Surf. B 112, 113-119 (2013).

27) Abdelrahim, M.E. Aerodynamic characteristics of nebulized terbutaline sulphate using the Andersen Cascade Impactor compared to the Next Generation Impactor. Pharm. Dev. Technol. 16, 137-145(2011).

28) Labiris, N.R.; Dolovich, M.B. Pulmonary drug delivery Part I: Physiological factors affecting therapeutic effectiveness of aerosolized medications. Br. J. Clin. Pharmacol. 56, 588-599(2003).

29) Prasad, D.; Chauhan, H.; Atef, E. Studying the effect of lipid chain length on the precipitation of a poorly water soluble drug from self-emulsifying drug delivery system on dispersion into aqueous medium. J. Pharm. Pharmacol. 65, 1134-44(2013).

30) Nesamony, J.; Kalra, A.; Majrad, M.S.; Boddu, S.H.S.; Jung, R.; Williams, F.E.; Schnapp, A.M.; Nauli, S.M.; Kalinoski, A.L. Development and characterization of nanostructured mists with potential for actively targeting poorly water-soluble compounds into the lungs. Pharm. Res. 30, 2625-2639 (2013).

31) Date, A.A.; Nagarsenker, M.S. Design and evaluation of self- nanoemulsifying drug delivery systems (SNEDDS) for cefpodoxime proxetil. Int. J. Pharm. 329, 166-172 (2007).

32) Kumar, G.P.; Rajeshwarrao, P. Nonionic surfactant vesicular systems for effective drug delivery-an overview. Acta Pharm. Sin B. 1, 208-219 (2011).

33) Lee, W.H.; Loo, C.Y.; Young, P.M. Recent advances in curcumin nanoformulation for cancer therapy. Expert Opin. Drug Deliv. 11, 1183-1201 (2014).

34) Ghazanfari, T.; Elhissi, A.M.A.; Ding, Z.; Taylor, K.M.G. The influence of fluid physicochemical properties on vibrating-mesh nebulization. Int. J. Pharm. 339, 103111 (2007).

35) Najlah, M.; Vali, A.; Taylor, M.; Arafat, B.T.; Ahmed, W.; Phoenix, D.A.; Elhissi, A. A study of the effects of sodium halides on the performance of air-jet and vibrating-mesh nebulizers. Int. J. Pharm. 456, 520-527 (2013). 